\title{
Modulation of Type-I Interferon Response by hsa-miR-374b-5p During Japanese Encephalitis Virus Infection in Human Microglial Cells
}

\author{
Meghana Rastogi and Sunit K. Singh* \\ Molecular Biology Unit, Institute of Medical Sciences, Banaras Hindu University, Varanasi, India
}

\section{OPEN ACCESS}

Edited by:

Darci R. Smith

Biological Defense Research Directorate, Naval Medical Research

Center, United States

Reviewed by:

Peter Halfmann,

University of Wisconsin-Madison,

United States

Bradley S. Hollidge,

United States Army Medical Research Institute of Infectious Diseases (USAMRIII), United States

*Correspondence: Sunit $K$. Singh sunitsingh2000@gmail.com

Specialty section:

This article was submitted to Virus and Host,

a section of the journal

Frontiers in Cellular and Infection Microbiology

Received: 09 May 2019

Accepted: 29 July 2019 Published: 09 August 2019

Citation:

Rastogi M and Singh SK (2019) Modulation of Type-I Interferon

Response by hsa-miR-374b-5p During Japanese Encephalitis Virus Infection in Human Microglial Cells. Front. Cell. Infect. Microbiol. 9:291.

doi: 10.3389/fcimb.2019.00291
Japanese Encephalitis virus (JEV) is a neurotropic ssRNA virus, belonging to the Flaviviridae family. JEV is one of the leading causes of the viral encephalitis in Southeast-Asian countries. JEV primarily infects neurons however, the microglial activation has been reported to further enhance the neuroinflammation and promote neuronal death. The PI3K/AKT pathway has been reported to play an important role in type-I interferon response via IRF3. Phosphatase and tensin homolog (PTEN), a negative regulator of PI3K/AKT pathway, participates in microglial polarization and neuroinflammation. The microRNAs are small non-coding endogenously expressed RNAs, which regulate the gene expression by binding at $3^{\prime}$ UTR of target gene. The human microglial cells were infected with JEV (JaOArS982 strain) and up-regulation of microRNA; hsa-miR-374b-5p was confirmed by qRT-PCR. The genes in PI3K/AKT pathway, over-expression and knock-down studies of hsa-miR-374b-5p with and without JEV infection were analyzed through immuno blotting. The regulatory role of hsa-miR-374b-5p on the expression of type-I interferon was determined by luciferase assays. JEV infection modulated the expression of hsa-miR-374b-5p and PI3K/AKT pathway via PTEN. The over-expression of hsa-miR-374b-5p suppressed the PTEN while up-regulated the AKT and IRF3 proteins, whereas, the knockdown rescued the PTEN expression and suppressed the AKT and IRF3 proteins. The modulation of hsa-miR-374b-5p regulated the type-I interferon response during JEV infection. In present study, we have shown the modulation of PTEN by hsa-miR-374b-5p, which regulated the PI3K/AKT/IRF3 axis in JEV infected microglial cells.

Keywords: JEV, human microglial cells, hsa-miR-374b-5p, type-I interferon response, viral immune evasion

\section{INTRODUCTION}

The Japanese Encephalitis virus (JEV) is a mosquito-borne Flavivirus belongs to Flaviviridae family. The Flaviviridae family also includes Dengue virus (DENV), West Nile Virus (WNV), Yellow Fever Virus (YFV), Tick Borne Encephalitis Virus (TBEV), and ZIKA Virus (ZIKV) (Gould and Solomon, 2008). JEV is one of the leading causes of viral encephalitis in Southeast-Asian countries, with an annual incidence of 70,000 and case fatality rate of $30-50 \%$, predominantly affecting children and elderly people (Campbell et al., 2011). JEV has devised several molecular strategies to evade the hosts immune response in order to establish itself successfully inside the host (Aleyas et al., 2010; Yang et al., 2011). 
Microglial cells are the resident macrophages in the central nervous system (CNS) and play roles in phagocytosis, immune surveillance, and antigen presentation (Colonna and Butovsky, 2017; Salter and Stevens, 2017). JEV has been reported to persist in microglial cells, which could be a plausible reservoir for infection (Thongtan et al., 2010). The JEV primarily infects and kills neurons and further enhances the neuroinflammatory events by producing chemokines and cytokines, which in turn activates microglial cells and leads to neuronal death in a bystander fashion (Ghoshal et al., 2007; Chen et al., 2010, 2012a). The microglial activation is regarded as one of the signs of neuroinflammation during viral infections (Conrady et al., 2013; Drokhlyansky et al., 2017; Mathur et al., 2017; Dello Russo et al., 2018). MicroRNAs are the endogenously expressed small non-coding RNAs, which regulate the gene expression by binding to the $3^{\prime}$ UTR of target mRNA (Ambros, 2004; Singh et al., 2008). We and others have previously reported the perturbation in cellular microRNA expression patterns during JEV infection for the purpose of replication and immune evasion (Cullen, 2013; Sharma et al., 2015, 2016; Rastogi et al., 2018).

The viral infection to microglial cells activates multiple signaling cascades, where the first-line-of-defense, type-I interferon response, plays a major role in orchestrating the anti-viral signaling by producing the IFN- $\alpha / \beta$ (Samuel, 2001; Randall and Goodbourn, 2008). The binding of these pleiotropic cytokines to their receptor IFNAR (IFN $\alpha$ R1/R2) stimulates the ISGs (IFN-stimulated genes) by binding to the IFN-stimulated response cis-elements (ISREs) inside the nucleus (Aaronson and Horvath, 2002). The type-I interferon responses have been reported to be transcriptionally regulated by RIG-I/Mda5 pathway, TLR3 and TLR4 mediated TRIF (Jiang et al., 2014) and TLR7/8 and TLR9 pathways. TRIF pathway leads to the activation of interferon regulatory factor 3 (IRF3), whereas the TLR7/8 and TLR9 pathway leads to the activation of interferon regulatory factor 7 (IRF7) (Daffis et al., 2008). We and others have reported the type I interferon promotes neuroinflammation in activated microglial cells (Furr and Marriot, 2012; Main et al., 2016) as well as in the JEV infected microglial cells (Manocha et al., 2014; Sharma et al., 2015; Lannes et al., 2017).

The involvement of the PI3K/AKT signaling has been reported in various cellular functions; however its dysregulation has been reported in neuroinflammation (Tang et al., 2017; Yang et al., 2017). In addition, the AKT/IRF3 axis has been studied in Traumatic Brain Injury (TBI) (Wang et al., 2015) and auto-inflammatory disorders (Oh et al., 2018). The activation of $\mathrm{PI} 3 \mathrm{~K} / \mathrm{AKT}$ pathway during viral infection has been correlated to viral replication, viral entry (Esfandiarei et al., 2004; Lee et al.,

\footnotetext{
Abbreviations: BSA, bovine serum albumin; DENV, Dengue virus; DMEM, Dulbecco's modified eagle medium; IFN- $\beta$, interferon $\beta$; IRF3 and 7 , interferon regulatory factor 3 and 7; ISGs, interferon stimulated genes; ISRE, IFNstimulated response elements; JEV, Japanese Encephalitis Virus; Mda5, Melanoma differentiation-associated protein 5; MOI, multiplicity of infection; p-PTEN, phosphorylated phosphatase and tensin homolog; PS, porcine kidney cells; PTEN, phosphatase and tensin homolog; PVDF, polyvinylidene fluoride; RIGI, retinoic acid inducible gene-I; TBEV, Tick Borne Encephalitis Virus; TLR3 and 4, Toll-like receptor 3 and 4; TRIF, TIR-domain-containing adapter-inducing interferon- $\beta$; WNV, West Nile Virus; YFV, Yellow Fever Virus; ZIKV, ZIKA virus.
}

2005) and virus-induced apoptosis (Esfandiarei et al., 2004; Lee et al., 2005; Schabbauer et al., 2008). In addition, the activation of IRF3 via PI3K/AKT pathway has been reported in the production of IFN- $\beta$ response (Joung et al., 2011; Lu et al., 2011; Tarassishin et al., 2011a; Cianciulli et al., 2016; Wang et al., 2017).

The PTEN (phosphatase and tensin homolog) is a tumor suppressor protein and a negative regulator for PI3K/AKT pathway, where the phosphorylation of PTEN negatively regulates PI3K/AKT pathway (Vazquez et al., 2000, 2001). The PTEN protein has been reported for its role in neuropathic pain, neuroinflammation, and modulating the microglial polarization through PTEN/AKT axis (Zhao et al., 2014; Huang et al., 2015; Wang et al., 2015; Cao et al., 2017). Although, PTEN has been studied in various tumors (Cheng et al., 2015; Yu et al., 2016), oncolytic viruses (Wu et al., 2017) but its role in microbial innate immunity has been recently identified, where it induces the interferon responses. The type-I interferon inducing capability of PTEN rely on its phosphatase activity, where the phosphorylation of PTEN activates the type-I interferon response (Li et al., 2016). The microRNA, hsa-miR-374b-5p has been identified in various cancers, neurodegeneration like Alzheimer's, hypoxic-ischemic encephalopathy and epilepsy etc. (Bian et al., 2019).

This is the first report where the role of PTEN has been highlighted in the modulation of type-I interferon response during JEV infection. We demonstrated the suppression of PTEN by microRNA, hsa-miR-374b-5p at $24 \mathrm{~h}$ of JEV infection in human microglial cells. The suppression of a negative regulator induced the PI3K/AKT pathway and promoted the type-I antiviral response via IRF3. The suppression of type-I anti-viral response during later stages of the infection process might be the strategy of JEV to subvert the anti-viral response.

\section{MATERIALS AND METHODS}

\section{Cell Culture}

The human microglial cells (Dello Russo et al., 2018), PS (porcine kidney cells) and Vero cells were cultured in DMEM (GIBCO) supplemented with heat inactivated 10\% FBS (GIBCO) and $100 \mathrm{U} / \mathrm{ml}$ of penicillin, $100 \mathrm{mg} / \mathrm{ml}$ streptomycin and 29.2 mg/ML. L-Glutamine (GIBCO) in humidified $\mathrm{CO}_{2}$ incubator at $37^{\circ} \mathrm{C}$. The human microglial cell line was the kind gift from Prof. Anirban Basu, National Brain Research Centre (NBRC), Manesar, Haryana.

\section{The Virus Propagation, Titration, and Infection}

The JaOArS982 strain of JEV was propagated in suckling BALB/c mice at NBRC, Manesar. The in-vitro propagation was done in the Vero cells at the MOI of 0.1 in the incomplete DMEM medium. The incomplete DMEM cell culture media was replaced by complete DMEM post infection $(2 \mathrm{~h})$ and left in $\mathrm{CO}_{2}$ incubator for 5 days or until $80 \%$ cell death was observed. The virus were titrated in PS cells by using plaque assay as described elsewhere (Sharma et al., 2015).

All of the JEV infection experiments were conducted in human microglial cells at the MOI of 5 in 6 well cell culture plates at the cell density of $0.3 \times 10^{6}$ cells/well in incomplete DMEM 
for $2 \mathrm{~h}$. The incomplete DMEM was changed to complete DMEM and the cells were harvested at 12,24, and $48 \mathrm{~h}$ post JEV infection and stored at $-80^{\circ} \mathrm{C}$ until further use.

\section{RNA Isolation, Micro RNA Expression and Real-Time PCR}

The Qiagen miRNeasy kit (\#217004; Qiagen, Venlo, Netherlands) was used for the isolation of total RNA from the microglial cells harvested at different time points. The complementary DNA (cDNA) was prepared by using Superscript II reverse transcriptase system (\#11904-018, Invitrogen, CS, USA) using the manufacturer's protocol. The thermal cycles for synthesizing cDNA were: $65^{\circ} \mathrm{C}-5 \mathrm{~min}, 25^{\circ} \mathrm{C}-10 \mathrm{~min}, 42^{\circ} \mathrm{C}-50 \mathrm{~min}$, and $70^{\circ} \mathrm{C}$ $10 \mathrm{~min}$, then, RNase $\mathrm{H}$ treatment $-20 \mathrm{~min}$ at $37^{\circ} \mathrm{C}$. The JEV infection in the human microglial cells was confirmed by qPCR against the JEV NS3 gene, normalized to GAPDH by using Agilent Brilliant III ultrafast SYBR green master mix (\#600882, Agilent Technologies, California, US) (Table 1).

To study the microRNA expression, the cDNA was synthesized by using MultiScribe TaqMan Reverse Transcriptase (\#4366596; Applied Biosystems, Waltham, MA, USA) along with hsa-miR-374b-5p specific primers according to manufacturer's protocol. The microRNA expression was analyzed using a real time PCR machine (Agilent AriaMx) by using a hsamiR-374b-5p-specific TaqMan probe and universal PCR master mix (\#4324018; Applied Biosystems). The expression of hsa-miR-374b-5p was normalized by endogenous control RNU6b expression.

\section{Protein Isolation, Estimation, and Immuno-Blotting}

The microglial cells harvested at different time points were used for protein isolation by using complete RIPA buffer ( $\# 89900$ Pierce, Thermo Fischer Scientific) and protease inhibitor cocktail (\#80650123 GE Biosciences). Briefly, the pellets were dissolved in RIPA buffer, sonicated for $5 \mathrm{~min}$ and centrifuged at 10,000 $\times \mathrm{g}$ for $20 \mathrm{~min}$ at $4^{\circ} \mathrm{C}$. The supernatant was collected, and the protein was estimated by BCA assay (\#23227 Pierce, Thermo Fischer Scientific). The protein was run on 10\% SDS-PAGE and then transferred to PVDF membrane (\#IPVH00010 Immobilon, Merck, Millipore). The blocking was done in 5\% skimmed milk (\#sc-2324 Santa Cruz) for an hour and the specific primary antibody was incubated in $5 \%$ skimmed milk at $4{ }^{\circ} \mathrm{C}$ overnight. The anti-PTEN antibody (\# 9559S CST), anti-AKT antibody (\#2920S CST), anti-p-AKT antibody (\#9271), anti-IRF3 antibody

TABLE 1 | List of primers, microRNA oligos, and scramble sequence.

\begin{tabular}{ll}
\hline Viral NS3 Forward & 5' AGAGCGGGGAAAAAGGTCAT 3' \\
\hline Viral NS3 Reverse & 5' 1 TTCACGCTCTTTCTACAGT 3' \\
GAPDH Forward & 5' ATGGGGGAAGGTGAAGGTCG 3' \\
GAPDH Reverse & 5' GGGGTCATTGATGGCAACAATA 3' \\
Mimics of hsa-miR-374b-5p & 5' AAAUGGCAUUAUAUAUAUUAUA 3' \\
Scramble of hsa-miR-374b-5p & 5' CAGAUUCUAUUUGCCCAAGAA 3'
\end{tabular}

(\#4302S CST), and anti- $\beta$-tubulin antibody (\# 250904 ABBiotec) were given in a 1:1000 dilution while anti-p-PTEN (\#9549P CST) and anti-p-IRF3 (\#4947S CST) were blocked and incubated in 5\% BSA, in 1:1000 dilutions overnight. The goat-anti-rabbit and mouse anti-goat secondary antibody were given at 1:50,000 for $2-3 \mathrm{~h}$ at room temperature in 5\% skimmed milk and 5\% BSA. The blots were developed in ChemiDoc (Azure Biosystems) by using west femto ECL substrate (\#34095 Super Signal West Femto Thermo Fischer Scientific) at different exposures. The Image J (ver: 1.42q) software has been used for densitometry of immunoblots.

\section{Over-expression and Inhibition Studies}

The human microglial cells were seeded in 6 well plate at the density of $0.4 \times 10^{6}$ cells/well, one day prior to transfection. The cells were transfected with 200 pmol of hsa-miR-374-5p mimics (Bioserve, Hyderabad, India) and 200 pmol of hsa-miR-374b$5 \mathrm{p}$ scramble (ILS) at the confluence of $70 \%$ by using chemical method, Lipofectamine 2000 (\#11668-019; Invitrogen, Carlsbad, CA, USA). The scramble sequence and mock transfected cells were used as controls. The overexpression study was confirmed by quantitative real-time PCR using TaqMan microRNA assay after $48 \mathrm{~h}$ post-transfection. The gene targets were confirmed by Immuno-blotting. The microRNA Inhibitor studies were performed by transfecting the anti-miR against (200 pmol) hsamiR-374b-5p (\#AM11339, Invitrogen, Carlsbad, CS, USA) and anti-miR scramble cy3 negative control (\#AM17011, Invitrogen, Carlsbad, CS, USA) by using Lipofectamine 2000 (\#11668019; Invitrogen, Carlsbad, CA, USA) and cells were harvested $48 \mathrm{~h}$ post transfection. The inhibitor studies were confirmed by quantitative real-time PCR using TaqMan microRNA assay. The gene targets were confirmed by Immuno-blotting. The transfected cells were infected post $24 \mathrm{~h}$ at the MOI of 5 for another $24 \mathrm{~h}$. The cells were harvested $48 \mathrm{~h}$ post-transfection.

\section{Luciferase Assay}

The human microglial cells were seeded in 6 well plates at the density of $0.4 \times 10^{6}$ cells/well and were co-transfected with IFN$\beta$-luciferase plasmid ( $1 \mu \mathrm{g})$ and normalized by $\beta$-gal (700 ng) vector. For over-expression and inhibitor studies, $200 \mathrm{pmol}$ of hsa-miR-374b-5p mimics, scramble, anti-miR and cy3 negative control were co-transfected in human microglial cells and the luciferase activity was measured post $48 \mathrm{~h}$. The cells were infected after $24 \mathrm{~h}$ post transfection at MOI 5.

The luminescence was measured by using a Luciferase assay kit (\#E4030; Promega, Madison, WI, USA) as per the manufacturer's protocol and the luminescence activity was measured on multimode reader (Synergy HTX, BioTek). The luminescence values were normalized by using $\beta$ gal plasmid vector. The $\beta$-galactosidase activity was measured at the absorbance of $420 \mathrm{~nm}$ by plate reader (imark plate reader, Bio-Rad).

\section{Statistical Analysis}

All experiments were conducted in triplicate $(n=3)$ and onetailed, paired Student's $t$-test was used to make comparison between data sets. The data are shown as mean \pm S.E from three 
independent experiments and data was considered significant when $P<0.05$; ${ }^{*}$ denotes $P<0.05$, ** denotes $P<0.01$, *** denotes $P<0.001$.

\section{RESULTS}

\section{JEV Infection Modulates the PI3K/AKT Pathway in Human Microglial Cells}

We and others have previously reported the JEV infection in the brain resident macrophages (microglial cells) (Thongtan et al., 2010; Manocha et al., 2014; Sharma et al., 2015; Lannes et al., 2017). The modulation of several cell signaling pathways leading to various neuroinflammatory events during JEV infection has been reported (Manocha et al., 2014; Sharma et al., 2015). The recent reports have highlighted the induction of immune responses through PI3K/AKT pathways (Sarkar et al., 2004; Hazeki et al., 2007; Polumuri et al., 2007; Radler et al., 2017) involving the AKT and IRF3 genes (Tarassishin et al., 2011b). PTEN is a negative regulator of PI3K/AKT pathway, and we observed the suppression of p-PTEN/PTEN at 12 and $24 \mathrm{~h}(60$ and $70 \%$ respectively) post JEV infection, whereas the expression of PTEN increased at $48 \mathrm{~h}$ compared to un-infected human microglial cells (Figures 1A,B). In addition, we demonstrated the modulation of AKT and IRF3 proteins at different time points by immunoblotting (Figures 1C-F). At early time point, 12 and $24 \mathrm{~h}$ post JEV infection, the $\mathrm{p}-\mathrm{AKT} / \mathrm{AKT}$ and $\mathrm{p}$-IRF3/IRF3 proteins were shown to be up-regulated, whereas at later stages of the infection progress (at $48 \mathrm{~h}$ ), the expression of p-AKT/AKT and p-IRF3/IRF3 have shown the decreasing trend (Figures 1C-F). Therefore, the JEV infection in human microglial cells modulates the PI3K/AKT pathway via PTEN during early and late courses of infection.

\section{JEV Infection in Human Microglial Cells Modulates the Micro RNA-374b-5p Expression}

The microRNAs have been reported to modulate the interferon response during viral infections (Buggele et al., 2013). In addition, hsa-miR-374b-5p has been reported to increase PTEN expression in various types of cancers (Li et al., 2015; Long et al., 2018). In our results, we demonstrated the gradual increase of microRNA hsa-miR-374b-5p expression levels from 24 to $48 \mathrm{~h}$ post JEV infection with the fold change of 2.5 to 5 (Figure 2). The up-regulation of microRNA hsa-miR-374b-5p at $24 \mathrm{~h}$ and suppression of PTEN at $24 \mathrm{~h}$ during JEV infection may be corelated (Figures 1A,B).

\section{The hsa-miR-374b-5p Targets PTEN and Modulates the Expression of AKT and IRF3}

To confirm the targeting of microRNA with PTEN, the hsamiR-374b-5p mimics and scrambled (hsa-miR-374b-5p) were transfected in human microglial cells and incubated for $48 \mathrm{~h}$ (Table 1). The up-regulation of mimics was confirmed by TaqMan microRNA assay, while no changes were observed in the samples transfected with scrambled sequences (Figure 3A). The expression of PTEN protein was reduced by $70 \%$ (Figures 3B,C), whereas the other proteins, AKT and IRF3 were up regulated by 1.5 and 1.2 -fold post transfection (Figures $3 \mathrm{D}-\mathbf{G}$ ). The microRNA, hsa-miR-374b-5p targeted to PTEN and modulated the genes involve in activation of interferon response via PI3K/AKT pathway.

\section{The anti-miR-374b-5p Rescues the Expression of PTEN and Suppresses the Expression of AKT and IRF3}

To further validate the targeting of microRNA, hsa-miR-374b$5 \mathrm{p}$ on its target PTEN, the hsa-miR-374b-5p was silenced by using microRNA inhibitors $(200 \mathrm{pM})$ and cy3 anti-miR negative control $(200 \mathrm{pM})$ in human microglial cells for $48 \mathrm{~h}$ utilizing transfection approaches. The suppression of microRNA was confirmed by TaqMan microRNA assay with around 60\% suppression, while no changes were observed in the cy 3 anti$\mathrm{miR}$ control (Figure 4A). The immunoblot analysis during the anti-miR treatment rescued the expression of PTEN by 1.1fold (Figures $4 \mathrm{~B}, \mathrm{C}$ ) and the expressions of AKT and IRF3 were suppressed by 20 and $60 \%$ post transfection (Figures $4 \mathrm{D}-\mathrm{G}$ ). Therefore, we concluded the hsa-miR-374b-5p alone can specifically modulate the expression of PI3K/AKT pathway.

\section{The Effect of hsa-miR-374b-5p Over-expression and Knockdown During JEV Infection}

The hsa-miR-374b-5p was overexpressed and knocked down in the microglial cells during the JEV infection to illustrate the role of microRNA. Since, the hsa-miR-374b-5p expression increased at $24 \mathrm{~h}$, the JEV infection was given $24 \mathrm{~h}$ post transfection at MOI 5 in human microglial cells. The infection was confirmed by NS3 q-PCR post $48 \mathrm{~h}$ of transfection (Figures 5A, 6A). The viral replication, as measured by the expression levels of NS3, increased during the over-expression of microRNA mimics, while it suppressed by inhibitors (Figures 5A, 6A). The effect of the hsa-miR-374b-5p mimics suppressed the p-PTEN/PTEN levels (Figures 5B,C) and upregulated the p-AKT/AKT and p-IRF3/IRF3 levels (Figures 5DG), whereas as a result of the suppression by anti-miR inhibitors, the expression levels were rescued for $\mathrm{p}$-PTEN/PTEN (Figures 6B,C) and suppressed the expression of p-AKT/AKT and p-IRF3/IRF3 levels (Figures 6D-G).

\section{The Activation of type-I Interferon Response During JEV Infection in Human Microglial Cells}

The phosphorylated form of AKT (p-AKT) activates IRF3 (Joung et al., 2011; Lu et al., 2011). The p-IRF3 in turn promote the type-I interferon response (Chang et al., 2006; Tarassishin et al., 2011a,b). To study the effect of hsa-miR-374b-5p on typeI interferon response during JEV infection, IFN- $\beta$ luciferase plasmid were co-transfected with microRNA mimics, scramble, anti-miR and cy 3 anti-miR negative control in human microglial cells for $48 \mathrm{~h}$. After, $24 \mathrm{~h}$ of transfection, JEV infection at MOI 5 was given for $24 \mathrm{~h}$. The result shows a 2 -fold up-regulation of IFN- $\beta$ response upon over-expression of the mimic (200 
A

p-PTEN

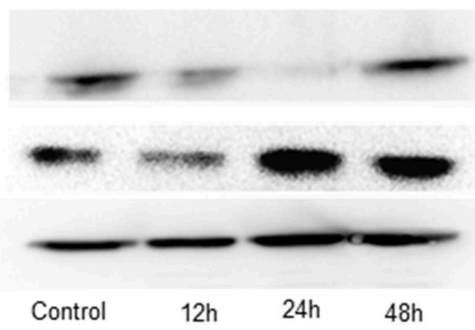

C

PTEN

$\beta$-tubulin

$$
\text { Control }
$$

$12 \mathrm{~h}$

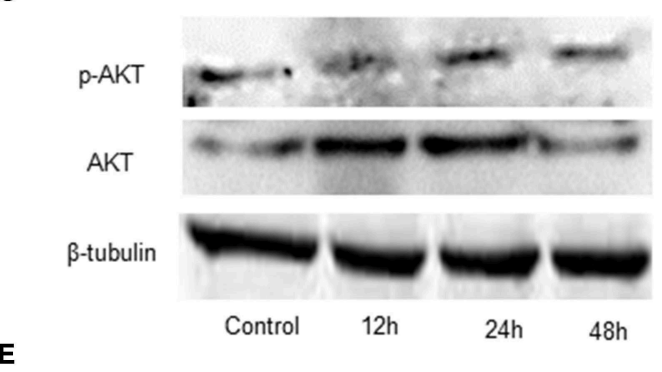

E

p-IRF3

IRF3

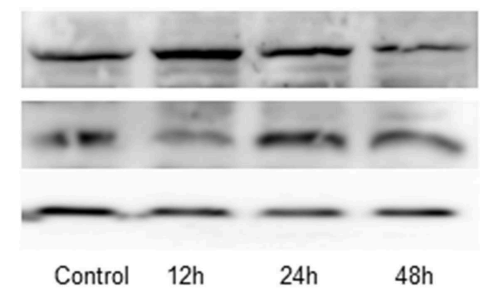

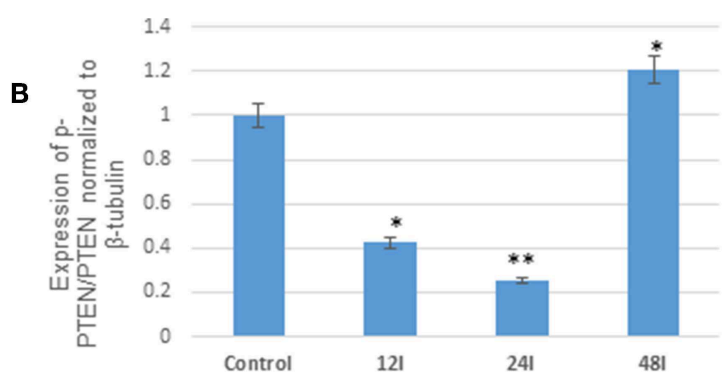

D
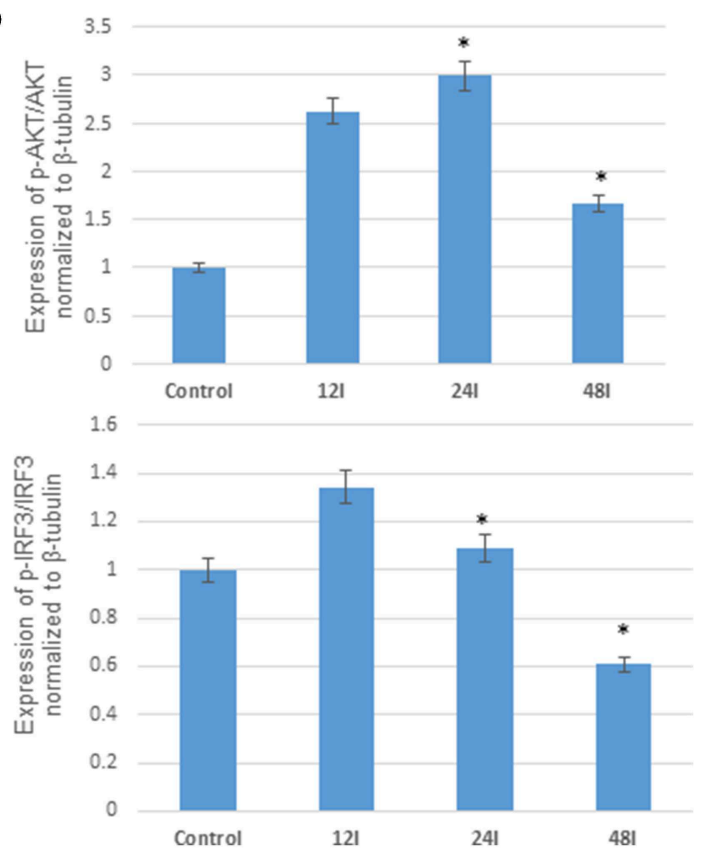

FIGURE 1 | JEV infection suppresses the negative regulator, PTEN and modulates PI3K/AKT pathway in human microglial cells. (A) Immunoblot of p-PTEN/PTEN at 12, 24, and 48 h post JEV infection compared to control (B) densitometry. (C) Immunoblot of p-AKT/AKT 12, 24, and 48 h post JEV infection compared to control (D) densitometry. (E) Immunoblot of p-IRF3/IRF3 12, 24, and $48 \mathrm{~h}$ post JEV infection compared to control (F) densitometry. All experiments were performed in triplicate $(n=3)$. The data are shown as mean \pm S.E from three independent experiments. The fold change is significant where * denotes $P<0.05$, ${ }^{\star *}$ denotes $P<0.01$,

*** denotes $P<0.001$.

pM) during JEV infection, while the inhibition of hsa-miR$374 \mathrm{~b}-5 \mathrm{p}$ using anti-miR, during JEV infection results in the $90 \%$ suppression. In addition, the type-I interferon response is mediated via hsa-miR-374b-5p, no JEV infection was given during over-expression and knock-down experiments ( $48 \mathrm{~h}$ ). The IFN- $\beta$ levels increase 1.1 -fold during mimic ( $200 \mathrm{pM})$ conditions. Similarly, the inhibition (anti-miR $200 \mathrm{pM}$ ) experiments suppress $40 \%$ of IFN- $\beta$ level which is less than the combination of JEV and inhibitor together (Figure 7).

\section{DISCUSSION}

The viral infection initiates an early innate anti-viral immune response by type-I interferon (IFN- $\alpha / \beta$ ) during the early phases of infection (Samuel, 2001; Randall and Goodbourn, 2008). The viral sensors in the cells induce the anti-viral responses through different pathways, such as the RIG-I and TLR3 pathways (Jiang et al., 2014), resulting in the production of type-I interferon. We and others earlier reported the modulation of interferon response during JEV infection (Manocha et al., 2014; Sharma et al., 2015; Ye et al., 2017). The JEV have devised several strategies to subvert the innate immune response in order to establish in host (Lee et al., 2005; Sharma et al., 2016). The PI3K/AKT pathway has been reported to play important roles in generation of immune responses either by positively or negatively regulating the downstream factors like, GSK3 $\beta$, AP1, NF-кB, NF-AT, CREB and JAK-STAT (Sarkar et al., 2004; Hazeki et al., 2007; Polumuri et al., 2007; Radler et al., 2017). In addition, the involvement of the PI3K/AKT pathway has also been reported in the expression of type I and type III interferon responses (Nguyen et al., 2001; Rani et al., 2002; Cianciulli et al., 2016). The activation of PI3K/AKT pathway results in the production of interferon response via IRF3 transcription factor (Tarassishin et al., 2011b; Cianciulli et al., 2016; Tang et al., 2017; Yang et al., 2017). 


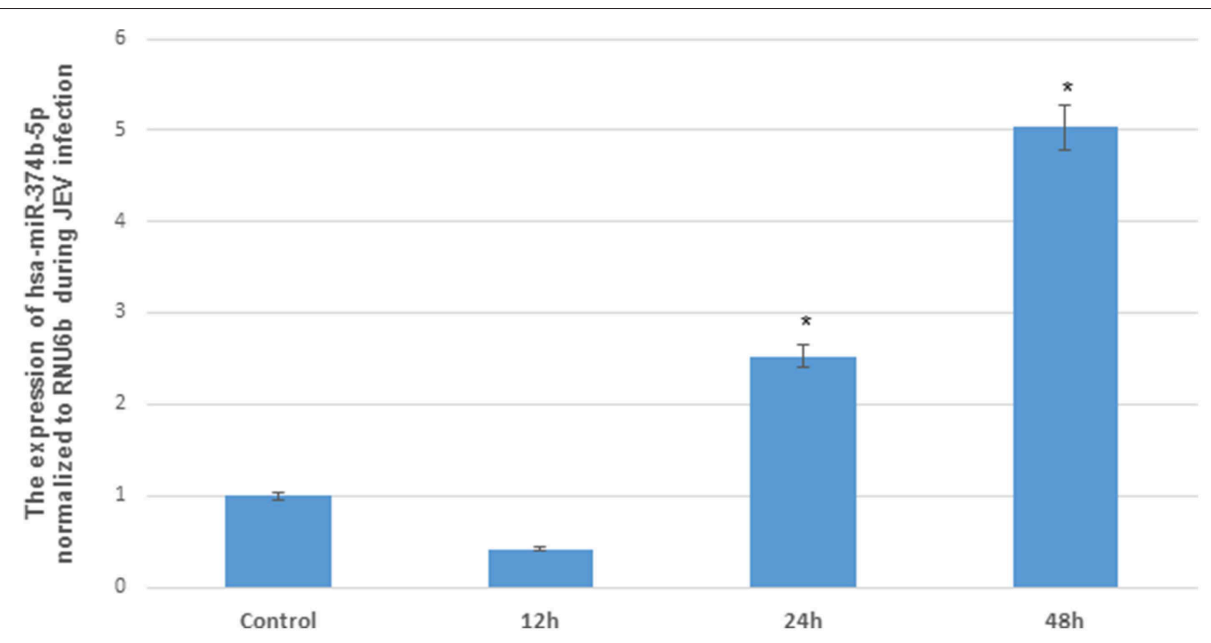

FIGURE 2 | The differential expression of hsa-miR-374b-5p after JEV (JaOARs982 strain) infection in human microglial cells. The change in hsa-miR-374b-5p after JEV (JaOARs982 strain) infection at different time points. The qRT-PCR by using TagMan microRNA assay shows the up-regulation from $24 \mathrm{~h}$ to $48 \mathrm{~h}$ by $2.5-5$-folds compared to control. The data are shown as mean \pm S.E from three independent experiments $(n=3)$. The fold change is significant where * denotes $P<0.05$, ${ }^{* *}$ denotes $P<0.01,{ }^{* * *}$ denotes $P<0.001$.

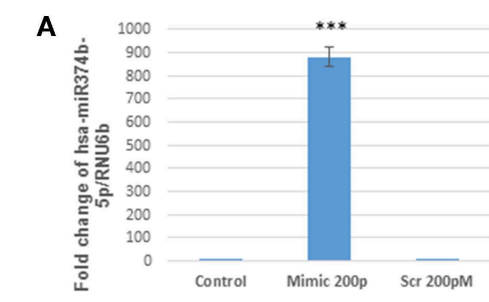

C

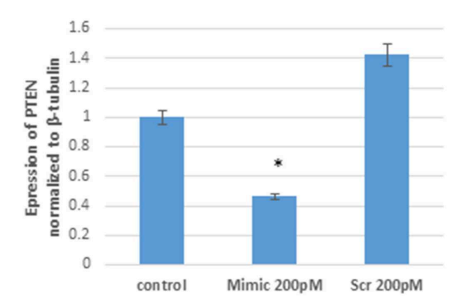

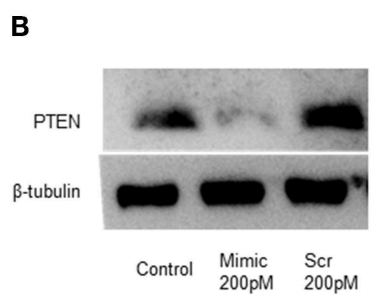

E

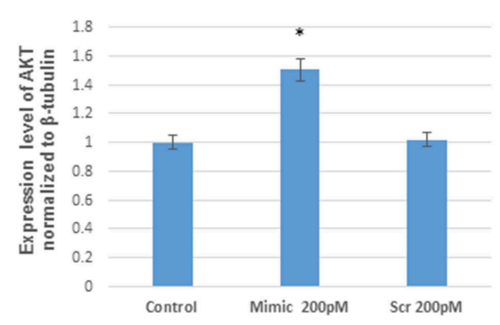

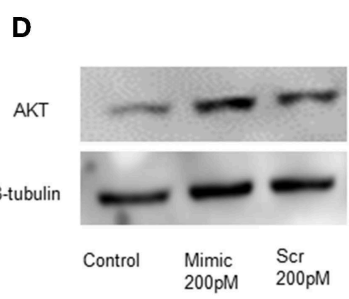

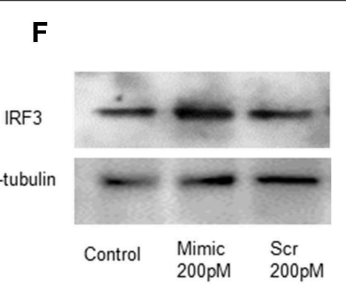

G

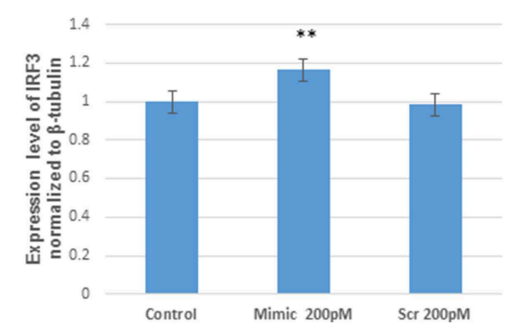

FIGURE 3 | The hsa-miR-374b-5p targets PTEN and activates the expression of AKT and IRF3. (A) qRT-PCR of hsa-miR-374b-5p overexpressed in human microglial cells by using RNA oligos at 200 pmol conc. (B) The immunoblots showing the suppression of PTEN by $70 \%$, $48 \mathrm{~h}$ post transfection. (C) The densitometry of PTEN. (D) The immunoblots showing up-regulation of AKT by 1.5-fold. (E) Densitometry of AKT. (F) The immunoblots showing up-regulation of IRF3 by 1.2-fold upon overexpression. (G) Densitometry of IRF3. The data are shown as mean \pm S.E from three independent experiments $(n=3)$. The fold change is significant where * denotes $P<0.05$, ${ }^{\star *}$ denotes $P<0.01$, ${ }^{\star \star *}$ denotes $P<0.001$.

The PTEN (Phosphatase and tensin homolog) is a dual phosphatase and a negative regulator for PI3K/AKT pathway. The PTEN protein is involved in myriad of cellular functions and its dysregulation has been reported in various types of cancers (Xiao et al., 2016; Khalid et al., 2017; Shen et al., 2019). However, many reports highlighted the aberrant expression of PTEN in neurodegenerative disorders, neuroinflammation, neuropathic pain, and microglial polarization (Ning et al., 2004; Choi et al., 2005; Griffin et al., 2005; Zhao et al., 2014; Wang et al., 2015; Cao et al., 2017). In addition, the microbes mediated-innate immune responses via PTEN have been recently reported (Li et al., 2016). The present study demonstrated the modulation of PTEN at different time points during JEV infection in human microglial cells. The dephosphorylated PTEN is an active form of PTEN, which suppressed the p-PTEN levels and led to the activation of PI3K/AKT pathway (Vazquez et al., 2001; Chen et al., 2012b; Zhao et al., 2014). The phosphorylation of AKT at ser473 led to the activation of interferon regulatory factor 3 (IRF3) by 


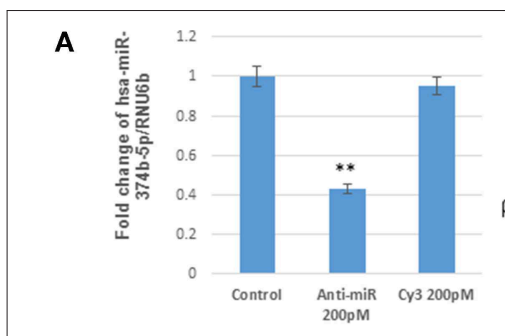

C

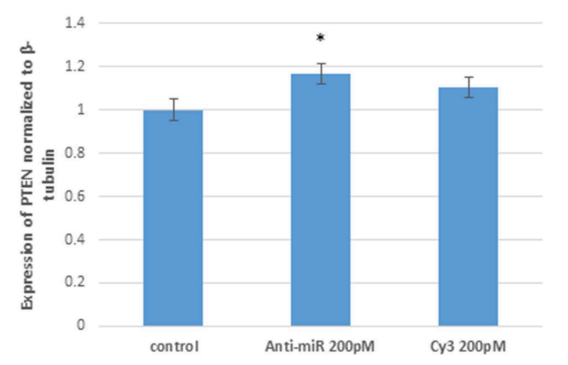

B

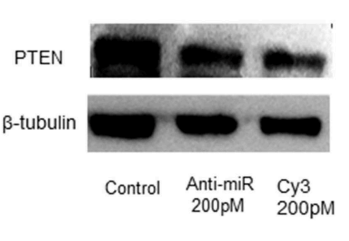

D

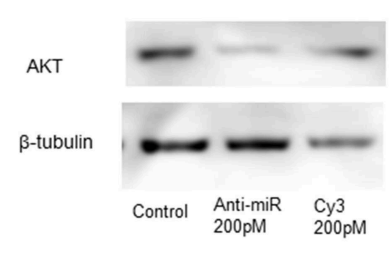

F

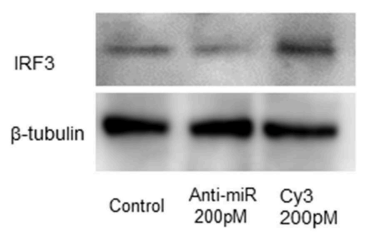

E

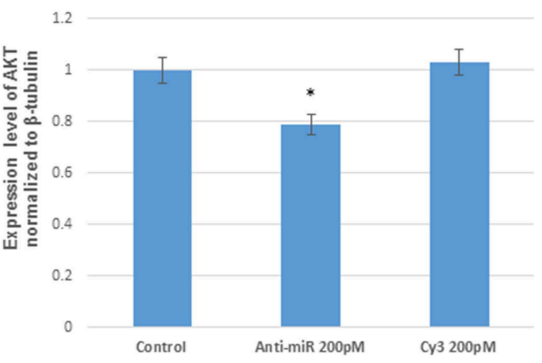

G

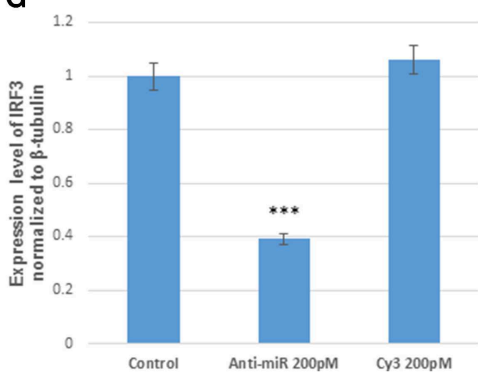

FIGURE 4 | The anti-miR-374b-5p rescues the expression of PTEN and suppresses the expression of AKT and IRF3. The knock down studies of hsa-miR-374b-5p (A) qRT-PCR knockdown of miR-374b-5p in human microglial cells by using inhibitors at 200 pmol conc. (B) The immuno blots showing the rescue of PTEN expression $48 \mathrm{~h}$ post transfection. (C) The densitometry of PTEN. (D) The immuno blots shows the suppression of AKT by $20 \%$ during transfection. (E) Densitometry of AKT. (F) The immuno blots shows the suppression of IRF3 by $60 \%$ during transfection. (G) Densitometry of IRF3. The data are shown as mean \pm S.E from three independent experiments $(n=3)$. The fold change is significant where ${ }^{*}$ denotes $P<0.05,{ }^{* \star}$ denotes $P<0.01,{ }^{* \star *}$ denotes $P<0.001$.

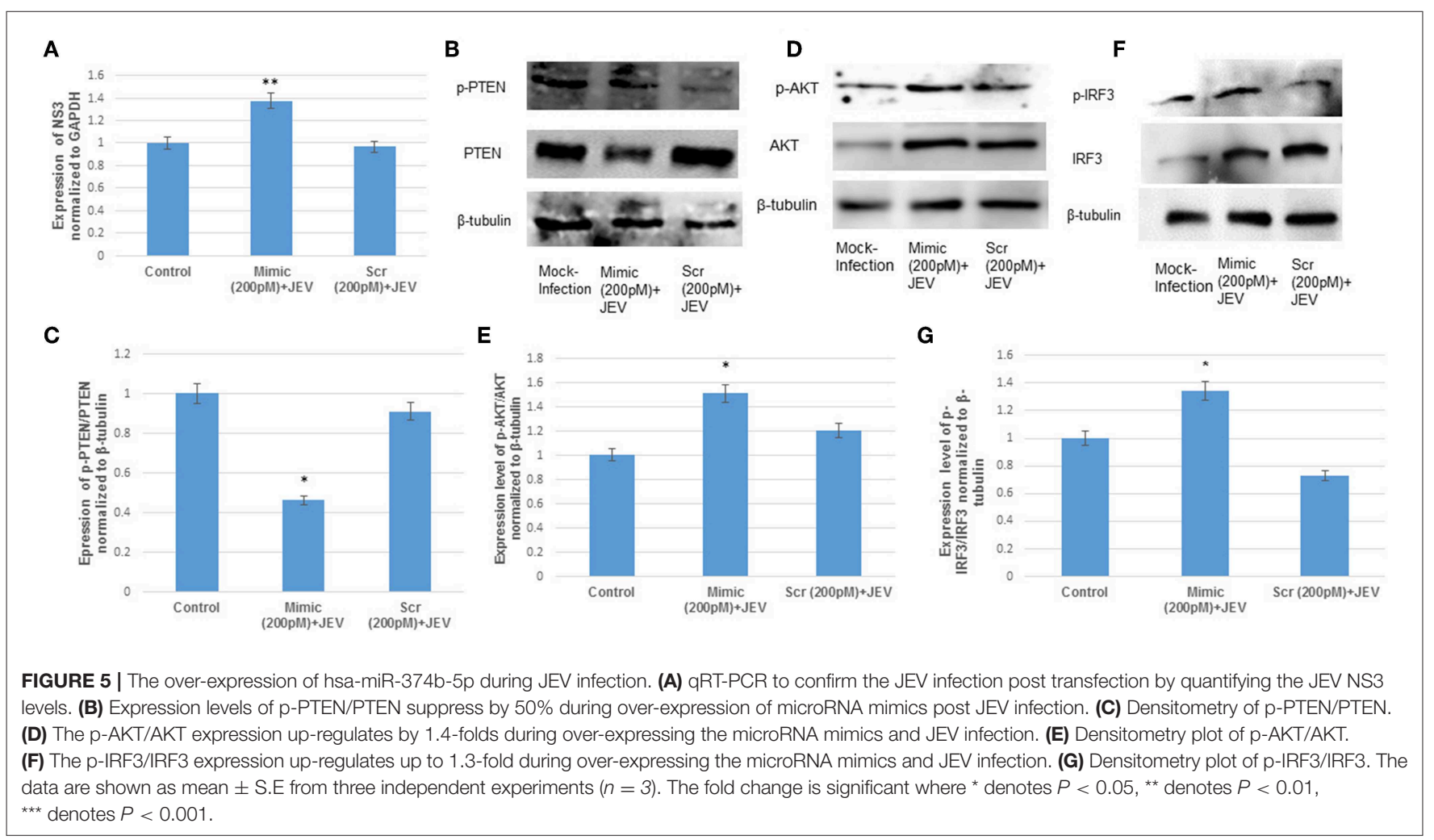

phosphorylating the IRF3 at ser396 (Tarassishin et al., 2011a,b). We earlier reported the generation of innate-antiviral immune response by a negative regulator, TRIM21 which resulted in the induction of type-I interferon via IRF3 activation (Manocha et al., 2014). A similar response has been shown by PTEN during JEV infection, where the suppression of PTEN resulted 

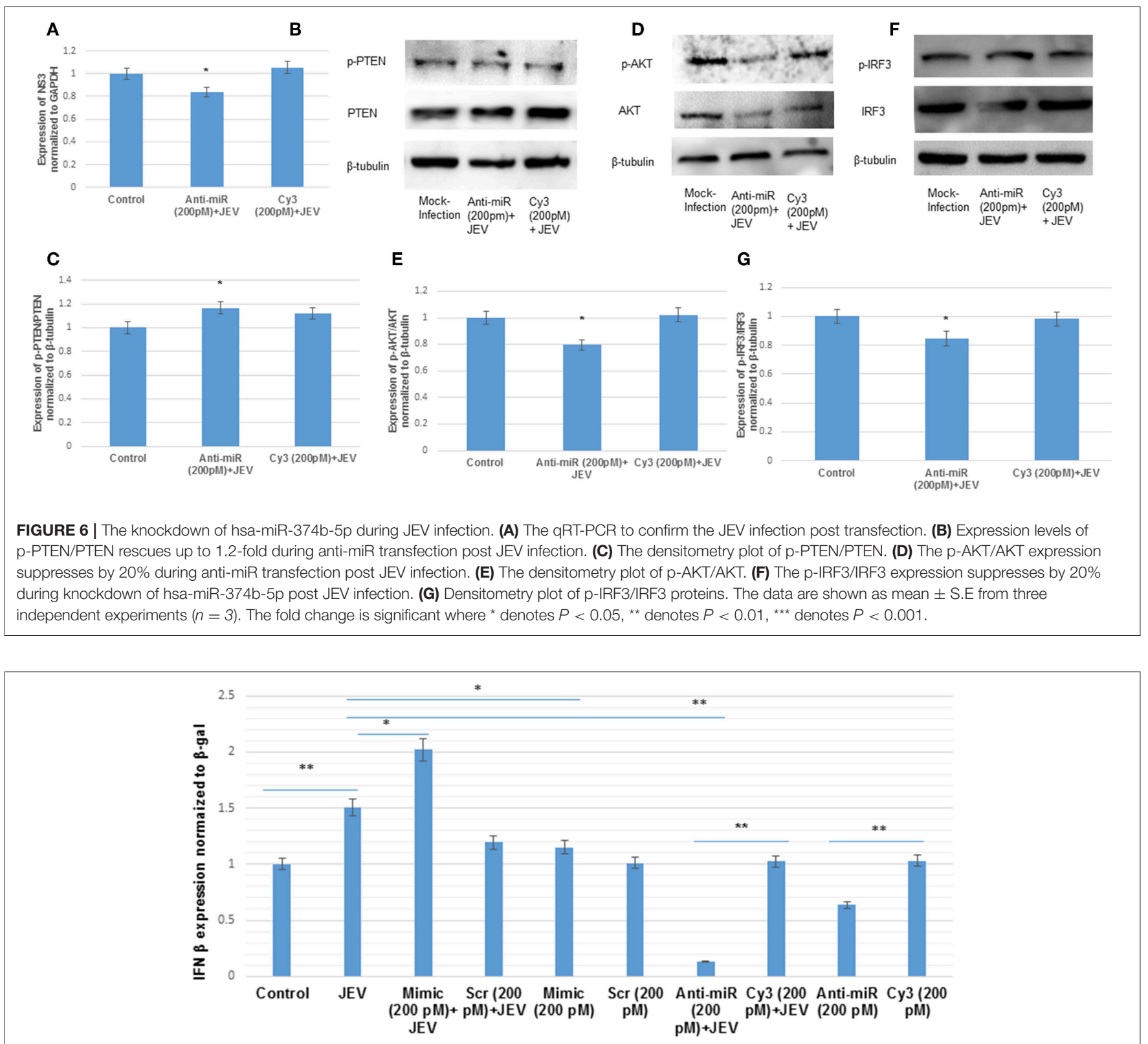

FIGURE 7 | The hsa-miR-374b-5p activates type-I interferon in JEV infected human microglial cells. The graph shows the up-regulation of IFN- $\beta$ level by 2 -fold during microRNA over-expression and JEV infection while the anti-miR during JEV infection suppresses the IFN- $\beta$ level by $90 \%$. The scramble and cy 3 anti-miR has been used as a control to further prove the specificity of microRNA, hsa-miR-374b-5p during JEV infection. In addition, the over-expression alone did increases the IFN- $\beta$ level by 1.1 -fold but it was comparatively lower than both the over-expression and JEV infection. The data are shown as mean \pm S.E from three independent experiments $(n=3)$. The fold change is significant where ${ }^{\star}$ denotes $P<0.05$, ${ }^{\star \star}$ denotes $P<0.01$, ${ }^{\star \star \star}$ denotes $P<0.001$.

in the activation of AKT proteins. The activated AKT, (p-AKT) phosphorylated the IRF3 expression (p-IRF3) at early time points during JE infection. The PI3K/AKT signaling pathway has been reported to be exploited by Flaviviruses in promoting viral entry, replication and blocking the apoptotic pathway (Lee et al., 2005; Das et al., 2010; Chen et al., 2017) in order to establish inside the host. On the other hand, the robust production of type-I interferon responses through the activation of PI3K/AKT pathway has been reported at early time point during microbial infections (Nguyen et al., 2001; Rani et al., 2002; Hazeki et al., 2007). The same has been observed in our previous study, where we have shown the increased levels of type-I interferon expression at the early time points of $12 \mathrm{~h}$ and $24 \mathrm{~h}$ during JEV infection (Manocha et al., 2014). Hence, the JEV modulated the p-PTEN/PTEN at an early time point to enter and replicate, while at the later stages of the infection's progression, the virus suppressed the PTEN and shut down the PI3K/AKT/IRF3 axis to suppress the type-I interferon response. 
MicroRNAs have been reported to be involved in the immune suppression during viral infections (Sharma et al., 2016; Rastogi et al., 2018). The hsa-miR-374b-5p has been extensively reviewed in various disorders, neurodegenerative disorders, encephalopathy, amyotrophic lateral sclerosis (ALS), and cardiovascular disorders (Bian et al., 2019). In this study we demonstrated the novel role of hsa-miR-374b-5p in immune activation during JEV infection. We demonstrated the upregulation of microRNA, hsa-miR-374b-5p during JEV infection $(24 \mathrm{~h})$. Further, the target prediction databases (TargetScan, miRdb, and miRWalk) identified PTEN as one of the putative targets. We demonstrated the targeting of the PTEN by microRNA, hsa-miR-374b-5p during JEV infection (24h) by utilizing over-expression and knock-down approaches. To further validate the involvement of AKT and IRF3 in type-I interferon activation, the expression levels of $\mathrm{p}-\mathrm{AKT} / \mathrm{AKT}$ and p-IRF3/IRF3 proteins were confirmed by immunoblotting. We observed the activation of the IFN- $\beta$ promoter during overexpression and suppression studies by using microRNA mimics and inhibitors, respectively, during the JEV infection (Figure 7). In addition, to delineate the effects of over-expression and knockdown, the IFN- $\beta$ levels were studied without JEV infection, where there was a comparatively lower expression of IFN- $\beta$ levels. Our findings have been complemented by the previously published reports, where the p-AKT was reported to activate IRF3, which resulted in the activation of type-I interferon responses (Rani et al., 2002; Randall and Goodbourn, 2008; Tarassishin et al., 2011b). In summary, we reported the modulation of PI3K/AKT/IRF3 axis by the negative regulator, PTEN via microRNA hsa-miR-374b-5p mediated pathway during JEV infection. However, the single microRNA can target multiple genes and the multiples genes can be targeted by multiple miRNAs or single miRNA. So, this PI3K/AKT/IRF3 axis might also get modulated by other microRNAs either by targeting the

\section{REFERENCES}

Aaronson, D. S., and Horvath, C. M. (2002). A road map for those who don't know JAK-STAT. Science 296, 1653-1655. doi: 10.1126/science.107 1545

Aleyas, A. G., Han, Y. W., George, J. A., Kim, B., Kim, K., Lee, C.-K., et al. (2010). Multifront assault on antigen presentation by Japanese encephalitis virus subverts CD8 ${ }^{+} \mathrm{T}$ cell responses. J. Immunol. 185, 1429-1441. doi: 10.4049/jimmunol.0902536

Ambros, V. (2004). The functions of animal microRNAs. Nature 431, 350-355. doi: $10.1038 /$ nature02871

Bian, H., Zhou, Y., Zhou, D., Zhang, Y., Shang, D., and Qi, J. (2019). The latest progress on miR-374 and its functional implications in physiological and pathological processes. J. Cell. Mol. Med. 23, 3063-3076. doi: $10.1111 / \mathrm{jcmm} .14219$

Buggele, W. A., Krause, K. E., and Horvath, C. M. (2013). Small RNA profiling of influenza A virus-infected cells identifies miR-449b as a regulator of histone deacetylase 1 and interferon beta. PLOS ONE 8:e76560. doi: 10.1371/journal.pone.0076560

Campbell, G. L., Hills, S. L., Fischer, M., Jacobson, J. A., Hoke, C. H., Hombach, J. M., et al. (2011). Estimated global incidence of Japanese encephalitis: a systematic review. Bull. World Health Organ. 89, 766-74; 774a-774e. doi: 10.2471/BLT.10.085233 same or other genes. Therefore, further studies are required to understand the involvement of other microRNAs in the modulation of PI3K/AKT/IRF3 axis during JEV infection. The activation of PI3K/AKT at early stages of the infection might be helpful for JEV internalization and the suppression at the later stages indicated the ability of JEV in modulating the host's cellular anti-viral response as a part of immune evasion during the course of infection.

\section{DATA AVAILABILITY}

The datasets generated for this study are available on request to the corresponding author.

\section{AUTHOR CONTRIBUTIONS}

MR conducted all of the experiments and wrote the manuscript. SS conceived the concept and guided throughout the experiments and contributed to the manuscript preparation.

\section{FUNDING}

Authors are highly acknowledging the funding support (Grant No: BT/PR8706/AGR/36/767/2013) from the Department of Biotechnology (DBT), Govt. of India. MR was a recipient of CSIR-Senior Research Fellowship.

\section{ACKNOWLEDGMENTS}

The authors highly acknowledge the help provided by Prof. Anirban Basu, National Brain Research Center, Manesar, Haryana. Authors are thankful to Prof. Adolfo Gracia-Sastre, Mount Sinai School of Medicine, New York, USA for providing IFN- $\beta$ Luciferase vector as a kind gift.

Cao, S. N., Yu, W. W., Zang, C. X., Bao, X. Q., Sun, H., and Zhang, D. (2017). Mechanism of non-receptor tyrosine kinase Src regulating neuroinflammation through phosphatase and tensin homology protein in microglia. Zhongguo Yi Xue Ke Xue Yuan Xue Bao 39, 534-538. doi: 10.3881/j.issn.1000-503X.2017.04.012

Chang, T. H., Liao, C. L., and Lin, Y. L. (2006). Flavivirus induces interferonbeta gene expression through a pathway involving RIG-I-dependent IRF3 and PI3K-dependent NF-кB activation. Microbes Infect. 8, 157-171. doi: 10.1016/j.micinf.2005.06.014

Chen, C. J., Ou, Y. C., Lin, S. Y., Raung, S. L., Liao, S. L., Lai, C. Y., et al. (2010). Glial activation involvement in neuronal death by Japanese encephalitis virus infection. J. Gen. Virol. 91, 1028-1037. doi: 10.1099/vir.0.013565-0

Chen, H. H., Chen, C. C., Lin, Y. S., Chang, P. C., Lu, Z. Y., Lin, C. F., et al. (2017). AR-12 suppresses dengue virus replication by down-regulation of PI3K/AKT and GRP78. Antiviral Res. 142, 158-168. doi: 10.1016/j.antiviral.2017. 02.015

Chen, S. T., Liu, R. S., Wu, M. F., Lin, Y. L., Chen, S. Y., Tan, D. T., et al. (2012a). CLEC5A regulates Japanese encephalitis virus-induced neuroinflammation and lethality. PLoS Pathog. 8:e1002655. doi: 10.1371/journal.ppat.1002655

Chen, Z., Chen, B., Xu, W. F., Liu, R. F., Yang, J., and Yu, C. X. (2012b). Effects of PTEN inhibition on regulation of tau phosphorylation in an okadaic acid-induced neurodegeneration model. Int. J. Dev. Neurosci. 30, 411-419. doi: 10.1016/j.ijdevneu.2012.08.003 
Cheng, D., Zhang, L., Yang, G., Zhao, L., Peng, F., Tian, Y., et al. (2015). Hepatitis C virus NS5A drives a PTEN-PI3K/Akt feedback loop to support cell survival. Liver Int. 35, 1682-1691. doi: 10.1111/liv.12733

Choi, J. S., Park, H. J., Kim, H. Y., Kim, S. Y., Lee, J. E., Choi, Y. S., et al. (2005). Phosphorylation of PTEN and Akt in astrocytes of the rat hippocampus following transient forebrain ischemia. Cell Tissue Res. 319, 359-366. doi: 10.1007/s00441-004-1033-0

Cianciulli, A., Calvello, R., Porro, C., Trotta, T., Salvatore, R., and Panaro, M. A. (2016). PI3k/Akt signalling pathway plays a crucial role in the anti-inflammatory effects of curcumin in LPS-activated microglia. Int. Immunopharmacol. 36, 282-290. doi: 10.1016/j.intimp.2016.05.007

Colonna, M., and Butovsky, O. (2017). Microglia function in the central nervous system during health and neurodegeneration. Annu. Rev. Immunol. 35, 441-468. doi: 10.1146/annurev-immunol-051116-052358

Conrady, C. D., Zheng, M., Van Rooijen, N., Drevets, D. A., Royer, D., Alleman, A., et al. (2013). Microglia and a functional type I IFN pathway are required to counter HSV-1-driven brain lateral ventricle enlargement and encephalitis. J. Immunol. 190, 2807-2817. doi: 10.4049/jimmunol.120 3265

Cullen, B. R. (2013). MicroRNAs as mediators of viral evasion of the immune system. Nat. Immunol. 14, 205-210. doi: 10.1038/ni.2537

Daffis, S., Samuel, M. A., Suthar, M. S., Keller, B. C., Gale, M., and Diamond, M. S. (2008). Interferon regulatory factor IRF-7 induces the antiviral alpha interferon response and protects against lethal West Nile virus infection. J. Virol. 82, 8465-8475. doi: 10.1128/JVI.00918-08

Das, S., Chakraborty, S., and Basu, A. (2010). Critical role of lipid rafts in virus entry and activation of phosphoinositide 3' kinase/Akt signaling during early stages of Japanese encephalitis virus infection in neural stem/progenitor cells. J. Neurochem. 115, 537-549. doi: 10.1111/j.1471-4159.2010. 06951.x

Dello Russo, C., Cappoli, N., Coletta, I., Mezzogori, D., Paciello, F., Pozzoli, G., et al. (2018). The human microglial HMC3 cell line: where do we stand? A systematic literature review. J. Neuroinflammation 15:259. doi: 10.1186/s12974-018-1288-0

Drokhlyansky, E., Goz Ayturk, D., Soh, T. K., Chrenek, R., O’loughlin, E., Madore, C., et al. (2017). The brain parenchyma has a type I interferon response that can limit virus spread. Proc. Natl. Acad. Sci. U.S.A. 114, E95-E104. doi: $10.1073 /$ pnas.1618157114

Esfandiarei, M., Luo, H., Yanagawa, B., Suarez, A., Dabiri, D., Zhang, J., et al. (2004). Protein kinase B/Akt regulates coxsackievirus B3 replication through a mechanism which is not caspase dependent. J. Virol. 78, 4289-4298. doi: 10.1128/JVI.78.8.4289-4298.2004

Furr, S. R., and Marriot, T. I. (2012). Viral CNS infections: role of glial pattern recognition receptors in neuroinflammation. Front. Microbiol. 3:201. doi: $10.3389 /$ fmicb. 2012.00201

Ghoshal, A., Das, S., Ghosh, S., Mishra, M. K., Sharma, V., Koli, P., et al. (2007). Proinflammatory mediators released by activated microglia induces neuronal death in Japanese encephalitis. Glia 55, 483-496. doi: 10.1002/glia.20474

Gould, E. A., and Solomon, T. (2008). Pathogenic flaviviruses. Lancet 371, 500-509. doi: 10.1016/S0140-6736(08)60238-X

Griffin, R. J., Moloney, A., Kelliher, M., Johnston, J. A., Ravid, R., Dockery, P., et al. (2005). Activation of Akt/PKB, increased phosphorylation of Akt substrates and loss and altered distribution of Akt and PTEN are features of Alzheimer's disease pathology. J. Neurochem. 93, 105-117. doi: 10.1111/j.1471-4159.2004.02949.x

Hazeki, K., Nigorikawa, K., and Hazeki, O. (2007). Role of phosphoinositide 3-kinase in innate immunity. Biol. Pharm. Bull. 30, 1617-1623. doi: $10.1248 / \mathrm{bpb} .30 .1617$

Huang, S. Y., Sung, C. S., Chen, W. F., Chen, C. H., Feng, C. W., Yang, S. N., et al. (2015). Involvement of phosphatase and tensin homolog deleted from chromosome 10 in rodent model of neuropathic pain. J. Neuroinflammation. 12:59. doi: 10.1186/s12974-015-0280-1

Jiang, R., Ye, J., Zhu, B., Song, Y., Chen, H., and Cao, S. (2014). Roles of TLR3 and RIG-I in mediating the inflammatory response in mouse microglia following Japanese encephalitis virus infection. J. Immunol. Res. 2014:787023. doi: 10.1155/2014/787023

Joung, S. M., Park, Z.-Y., Rani, S., Takeuchi, O., Akira, S., and Lee, J. Y. (2011). Akt contributes to activation of the TRIF-dependent signaling pathways of
TLRs by interacting with TANK-binding kinase 1. J. Immunol. 186, 499-507. doi: 10.4049/jimmunol.0903534

Khalid, A., Hussain, T., Manzoor, S., Saalim, M., and Khaliq, S. (2017). PTEN: a potential prognostic marker in virus-induced hepatocellular carcinoma. Tumour Biol. 39:1010428317705754. doi: 10.1177/1010428317705754

Lannes, N., Summerfield, A., and Filgueira, L. (2017). Regulation of inflammation in Japanese encephalitis. J. Neuroinflammation 14:158. doi: 10.1186/s12974-017-0931-5

Lee, C. J., Liao, C. L., and Lin, Y. L. (2005). Flavivirus activates phosphatidylinositol 3-kinase signaling to block caspase-dependent apoptotic cell death at the early stage of virus infection. J. Virol. 79, 8388-8399. doi: 10.1128/JVI.79.13.8388-8399.2005

Li, N., Yang, L., Wang, H., Yi, T., Jia, X., Chen, C., et al. (2015). MiR-130a and MiR-374a function as novel regulators of cisplatin resistance in human ovarian cancer A2780 cells. PLoS ONE 10:e0128886. doi: 10.1371/journal.pone.0128886

Li, S., Zhu, M., Pan, R., Fang, T., Cao, Y. Y., Chen, S., et al. (2016). The tumor suppressor PTEN has a critical role in antiviral innate immunity. Nat. Immunol. 17, 241-249. doi: 10.1038/ni.3311

Long, Z. W., Wu, J. H., Cai, H., Wang, Y. N., and Zhou, Y. (2018). MiR$374 \mathrm{~b}$ promotes proliferation and inhibits apoptosis of human GIST cells by inhibiting PTEN through activation of the PI3K/Akt pathway. Mol. Cells 41, 532-544. doi: 10.14348/molcells.2018.2211

Lu, X., Masic, A., Liu, Q., and Zhou, Y. (2011). Regulation of influenza A virus induced CXCL-10 gene expression requires PI3K/Akt pathway and IRF3 transcription factor. Mol. Immunol. 48, 1417-1423. doi: 10.1016/j.molimm.2011.03.017

Main, B. S., Zhang, M., Brody, K. M., Ayton, S., Frugier, T., Steer, D., et al. (2016). Type-1 interferons contribute to the neuroinflammatory response and disease progression of the MPTP mouse model of Parkinson's disease. Glia 64, 1590-1604. doi: 10.1002/glia.23028

Manocha, G. D., Mishra, R., Sharma, N., Kumawat, K. L., Basu, A., and Singh, S. K. (2014). Regulatory role of TRIM21 in the type-I interferon pathway in Japanese encephalitis virus-infected human microglial cells. J. Neuroinflammation 11:24. doi: 10.1186/1742-2094-11-24

Mathur, V., Burai, R., Vest, R. T., Bonanno, L. N., Lehallier, B., Zardeneta, M. E., et al. (2017). Activation of the STING-dependent type I interferon response reduces microglial reactivity and neuroinflammation. Neuron 96, 1290-1302.e6. doi: 10.1016/j.neuron.2017.11.032

Nguyen, H., Ramana, C. V., Bayes, J., and Stark, G. R. (2001). Roles of phosphatidylinositol 3-kinase in interferon-gamma-dependent phosphorylation of STAT1 on serine 727 and activation of gene expression. J. Biol. Chem. 276, 33361-33368. doi: 10.1074/jbc.M105070200

Ning, K., Pei, L., Liao, M., Liu, B., Zhang, Y., Jiang, W., et al. (2004). Dual neuroprotective signaling mediated by downregulating two distinct phosphatase activities of PTEN. J. Neurosci. 24, 4052-4060. doi: 10.1523/JNEUROSCI.5449-03.2004

Oh, C., Ryoo, J., Park, K., Kim, B., Daly, M. B., Cho, D., et al. (2018). A central role for PI3K-AKT signaling pathway in linking SAMHD1-deficiency to the type I interferon signature. Sci. Rep. 8:84. doi: 10.1038/s41598-017-18308-8

Polumuri, S. K., Toshchakov, V. Y., and Vogel, S. N. (2007). Role of phosphatidylinositol-3 kinase in transcriptional regulation of TLR-induced IL-12 and IL-10 by Fc gamma receptor ligation in murine macrophages. J. Immunol. 179, 236-246. doi: 10.4049/jimmunol.179.1.236

Radler, P. D., Wehde, B. L., and Wagner, K. U. (2017). Crosstalk between STAT5 activation and PI3K/AKT functions in normal and transformed mammary epithelial cells. Mol. Cell Endocrinol. 451, 31-39. doi: 10.1016/j.mce.2017.04.025

Randall, R. E., and Goodbourn, S. (2008). Interferons and viruses: an interplay between induction, signalling, antiviral responses and virus countermeasures. J. Gen. Virol. 89, 1-47. doi: 10.1099/vir.0.83391-0

Rani, M. R., Hibbert, L., Sizemore, N., Stark, G. R., and Ransohoff, R. M. (2002). Requirement of phosphoinositide 3-kinase and Akt for interferonbeta-mediated induction of the beta-R1 (SCYB11) gene. J. Biol. Chem. 277, 38456-38461. doi: 10.1074/jbc.M203204200

Rastogi, M., Srivastava, N., and Singh, S. K. (2018). Exploitation of microRNAs by Japanese Encephalitis virus in human microglial cells. J. Med. Virol. 90, 648-654. doi: 10.1002/jmv.24995

Salter, M. W., and Stevens, B. (2017). Microglia emerge as central players in brain disease. Nat. Med. 23, 1018-1027. doi: 10.1038/nm.4397 
Samuel, C. E. (2001). Antiviral actions of interferons. Clin. Microbiol. Rev. 14, 778-809, table of contents. doi: 10.1128/CMR.14.4.778-809.2001

Sarkar, S. N., Peters, K. L., Elco, C. P., Sakamoto, S., Pal, S., and Sen, G. C. (2004). Novel roles of TLR3 tyrosine phosphorylation and PI3 kinase in double-stranded RNA signaling. Nat. Struct. Mol. Biol. 11, 1060-1067. doi: $10.1038 / \mathrm{nsmb} 847$

Schabbauer, G., Luyendyk, J., Crozat, K., Jiang, Z., Mackman, N., Bahram, S., et al. (2008). TLR4/CD14-mediated PI3K activation is an essential component of interferon-dependent VSV resistance in macrophages. Mol. Immunol. 45, 2790-2796. doi: 10.1016/j.molimm.2008.02.001

Sharma, N., Kumawat, K. L., Rastogi, M., Basu, A., and Singh, S. K. (2016). Japanese Encephalitis Virus exploits the microRNA-432 to regulate the expression of suppressor of cytokine signaling (SOCS) 5. Sci. Rep. 6, 27685-27685. doi: $10.1038 /$ srep 27685

Sharma, N., Verma, R., Kumawat, K. L., Basu, A., and Singh, S. K. (2015). miR146a suppresses cellular immune response during Japanese encephalitis virus JaOArS982 strain infection in human microglial cells. J. Neuroinflammation 12:30. doi: 10.1186/s12974-015-0249-0

Shen, F., Zheng, H., Zhou, L., Li, W., Liu, J., and Xu, X. (2019). Identification of CD28 and PTEN as novel prognostic markers for cervical cancer. J. Cell Physiol. 234, 7004-7011. doi: $10.1002 /$ jcp. 27453

Singh, S. K., Pal Bhadra, M., Girschick, H. J., and Bhadra, U. (2008). MicroRNAs-micro in size but macro in function. FEBS J. 275, 4929-4944. doi: $10.1111 / j .1742-4658.2008 .06624 . x$

Tang, G., Yang, H., Chen, J., Shi, M., Ge, L., Ge, X., et al. (2017). Metformin ameliorates sepsis-induced brain injury by inhibiting apoptosis, oxidative stress and neuroinflammation via the PI3K/Akt signaling pathway. Oncotarget 8, 97977-97989. doi: 10.18632/oncotarget.20105

Tarassishin, L., Loudig, O., Bauman, A., Shafit-Zagardo, B., Suh, H. S., and Lee, S. C. (2011a). Interferon regulatory factor 3 inhibits astrocyte inflammatory gene expression through suppression of the proinflammatory miR-155 and miR-155*. Glia 59, 1911-1922. doi: 10.1002/glia.21233

Tarassishin, L., Suh, H. S., and Lee, S. C. (2011b). Interferon regulatory factor 3 plays an anti-inflammatory role in microglia by activating the PI3K/Akt pathway. J. Neuroinflammation 8:187. doi: 10.1186/1742-2094-8-187

Thongtan, T., Cheepsunthorn, P., Chaiworakul, V., Rattanarungsan, C., Wikan, N., and Smith, D. R. (2010). Highly permissive infection of microglial cells by Japanese encephalitis virus: a possible role as a viral reservoir. Microbes Infect. 12, 37-45. doi: 10.1016/j.micinf.2009.09.013

Vazquez, F., Grossman, S. R., Takahashi, Y., Rokas, M. V., Nakamura, N., and Sellers, W. R. (2001). Phosphorylation of the PTEN tail acts as an inhibitory switch by preventing its recruitment into a protein complex. J. Biol. Chem. 276, 48627-48630. doi: 10.1074/jbc.C100556200

Vazquez, F., Ramaswamy, S., Nakamura, N., and Sellers, W. R. (2000). Phosphorylation of the PTEN tail regulates protein stabilityand function. Mol. Cell Biol. 20, 5010-5018. doi: 10.1128/MCB.20.14.5010-50 18.2000

Wang, G., Shi, Y., Jiang, X., Leak, R. K., Hu, X., Wu, Y., et al. (2015). HDAC inhibition prevents white matter injury by modulating microglia/macrophage polarization through the GSK3beta/PTEN/Akt axis. Proc. Natl. Acad. Sci. U.S.A. 112, 2853-2858. doi: 10.1073/pnas.1501441112

Wang, L., Yang, L., Fikrig, E., and Wang, P. (2017). An essential role of PI3K in the control of West Nile virus infection. Sci. Rep. 7:3724. doi: 10.1038/s41598-017-03912-5

Wu, Q., Li, Z., Mellor, P., Zhou, Y., Anderson, D. H., and Liu, Q. (2017). The role of PTEN - HCV core interaction in hepatitis C virus replication. Sci. Rep. 7:3695. doi: 10.1038/s41598-017-03052-w

Xiao, J., Hu, C. P., He, B. X., Chen, X., Lu, X. X., Xie, M. X., et al. (2016). PTEN expression is a prognostic marker for patients with non-small cell lung cancer: a systematic review and meta-analysis of the literature. Oncotarget 7 , 57832-57840. doi: 10.18632/oncotarget.11068

Yang, Y., Liu, Y., Xue, J., Yang, Z., Shi, Y., Shi, Y., et al. (2017). MicroRNA-141 targets Sirtl and inhibits autophagy to reduce HBV replication. Cell Physiol. Biochem. 41, 310-322. doi: 10.1159/000456162

Yang, Y., Ye, J., Yang, X., Jiang, R., Chen, H., and Cao, S. (2011). Japanese encephalitis virus infection induces changes of mRNA profile of mouse spleen and brain. Virol. J. 8:80. doi: 10.1186/1743-422X-8-80

Ye, J., Chen, Z., Li, Y., Zhao, Z., He, W., Zohaib, A., et al. (2017). Japanese encephalitis virus NS5 inhibits type I interferon (IFN) production by blocking the nuclear translocation of IFN regulatory factor 3 and NF-KB. J. Virol. 91, e00039-e00017. doi: 10.1128/JVI.00039-17

Yu, X., Li, T., Xia, Y., Lei, J., Wang, Y., and Zhang, L. (2016). Herpes simplex virus type 1 VP22-mediated intercellular delivery of PTEN increases the antitumor activity of PTEN in esophageal squamous cell carcinoma cells in vitro and in vivo. Oncol. Rep. 35, 3034-3040. doi: 10.3892/or.2016.4694

Zhao, M., Zhou, A., Xu, L., and Zhang, X. (2014). The role of TLR4-mediated PTEN/PI3K/AKT/NF- $\mathrm{B}$ signaling pathway in neuroinflammation in hippocampal neurons. Neuroscience 269, 93-101. doi: 10.1016/j.neuroscience.2014.03.039

Conflict of Interest Statement: The authors declare that the research was conducted in the absence of any commercial or financial relationships that could be construed as a potential conflict of interest.

Copyright (๑) 2019 Rastogi and Singh. This is an open-access article distributed under the terms of the Creative Commons Attribution License (CC BY). The use, distribution or reproduction in other forums is permitted, provided the original author(s) and the copyright owner(s) are credited and that the original publication in this journal is cited, in accordance with accepted academic practice. No use, distribution or reproduction is permitted which does not comply with these terms. 